\title{
Factores que Afectan las Decisiones de Compra de Tilapia (Oreochromis Ssp.) en Consumidores del Gran Santiago
}

\author{
Luis Sáez T ${ }^{\mathbf{1}}$; Michael Cancino F $^{\mathbf{1}}$; Carlos Díaz $\mathbf{R}^{\mathbf{1}}$.; Mario Maino M. ${ }^{\mathbf{2}}$ \\ ${ }^{1}$ Departamento de Gestión Agraria, Universidad de Santiago de Chile. Avenida Ecuador 3769, Estación \\ Central, Santiago, Chile. \\ ${ }^{2}$ Facultad de Ciencias Veterinarias, Universidad de Chile. Avenida Santa Rosa 11735, La Pintana, Santiago, \\ Chile. \\ Email: luis.saez@usach.cl
}

\begin{abstract}
Resumen
La evaluación de factores que afectan la compra de nuevos productos alimenticios, tales como la Tilapia (Oreochromis spp), por medio de la evaluación exploratoria de motivaciones de compra, atributos de calidad relevantes en el momento de compra, seguido de evaluación sensorial por parte de consumidores, ayuda a estudiar el comportamiento de los consumidores frente a productos desconocidos y sus atributos de calidad. Para estudiar los factores que motivan la compra de Tilapia y en general de pescado en consumidores del Gran Santiago, se recurrió a un estudio exploratorio por medio de entrevistas en profundidad, seguido de una encuesta a una muestra de 384 personas. Posterior a ello se realizó un estudio sensorial por medio de una panel piloto, con panelistas no entrenados. Los resultados sugieren que las motivaciones de compra son similares en Tilapia, así como para otras variedades de pescados y si bien, los consumidores declaran inicialmente no estar motivados a comprar una variedad de pescado desconocida, los resultados del estudio sensorial muestran un nivel de aceptación alto de atributos tales como sabor, color y aroma, de acuerdo a una escala hedónica de 5 puntos, no obstante la valoración de dichos atributos sería dependiente del tipo de cocción al que se somete el producto.
\end{abstract}

\section{Introducción}

Un producto que desde el año 2007 ha cobrado notoriedad a causa del alto aumento de su consumo en Estados Unidos, y que generó el progresivo aumento en su producción, en países de América Latina, es la Tilapia (Oreochromis spp.). Esta especie es originaria de África y el Cercano Oriente, y es cultivado en aproximadamente 85 países en el mundo. La primera variedad de Tilapia (Oreochromis Spp.) empleada para producción acuícola fue introducida en Singapur en 1946 la que tiene una coloración negra y se conoce con el nombre científico Oreochromis mossambicus (Castillo, 2005).
Hay más de 70 especies y 100 subespecies de Tilapias las que han sido agrupadas en cuatro géneros de acuerdo con sus hábitos reproductivos, como Oreochromis (Gunther), Tilapia (Smith), Sarotherodon (Rupell), Danakilia (Thys). En la década de los 80 la Tilapia Roja logró acelerar la piscicultura comercial, siendo introducida en 1982 a Colombia, 1989 a Venezuela y en 1993 a Ecuador junto a otros países Centroamericanos, Caribeños y Norteamericanos (Castillo, 2005).

Los precios de venta han variado bastante, según lo expuesto en la Tabla 4, el precio del filete fresco, en el 
año 1992 su precio era de US\$5,04 el kilo (FOB), alcanzando US\$6,04 en el año 2005. Los precios en el caso del filete congelado no han tenido variaciones significativas, de US\$3,2 en 1992 a US\$3,6 en el 2005 (Castillo, 2005). En el año 2010 el precio medio del filete de Tilapia era de US\$7 el kilo (FOB) y el de filete de Tilapia congelada en US\$3,7 el kilo (Castillo, 2010).

El principal productor a nivel mundial es China, que concentra un $45 \%$ de las exportaciones mundiales de filete de Tilapia fresco y Tilapia congelada, mientras que Estados Unidos es el principal importador mundial, con 96\% del volumen de las importaciones mundiales. (CORPEI, 2005).

De acuerdo a los datos de CORPEI (2005) los principales factores que motivan el consumo de la Tilapia en Estados Unidos son los beneficios para la salud del consumo de pescado y alimentos del mar, la frescura y cualidades del pescado, disponibilidad y precio consistentes durante todo el año, precios convenientes, la durabilidad del pescado fresco, demanda de conveniencia, preferencias del sector institucional. Según proyecciones, el consumo de la Tilapia en EE.UU aumentará. Por otra parte, en la Unión Europea los principales consumidores de Tilapia son el Reino Unido, Francia y Bélgica, donde se comercializa mayormente en forma congelada $(95 \%$ del total). En el caso de Tilapia fresca, Zimbabwe es el principal proveedor de este mercado, especialmente con destino al Reino Unido. Por el contrario, América Latina tiene una presencia muy pequeña en este mercado; el precio de esta presentación es de alrededor de 7 euros por kilogramos. (Maradiegue et. al 2005).

Si bien la Tilapia fue considerada en otro tiempo un pescado de bajo valor, adecuada solamente para mercados étnicos, este producto ha ganado popularidad en años recientes. Actualmente, tiene buena aceptación por el consumidor y es considerada una atractiva opción del menú en cadenas de restaurantes a nivel nacional e internacional (Morales et al, 2004), y en el caso de Chile, actualmente, es posible encontrar tilapia fresca y congelada en diferentes supermercados del Gran Santiago y eventualmente de regiones, no obstante no existen estudios previos relativos a los gustos y preferencias de los consumidores de esta ciudad, sino que más bien se enfoca a los aspectos productivos del cultivo de este recurso acuícola. Debido a lo anterior, el presente estudio busca proporcionar información relativa a los factores que podrían motivar el consumo de pescado entre consumidores del Gran Santiago y en particular de Tilapia (Oreochromis spp) y evaluar la aceptabilidad a nivel de atributos sensoriales de diferentes formas de preparación de este producto.

\section{Materiales y métodos}

Se realizaron seis entrevistas en profundidad durante Septiembre de 2010. Cada entrevista fue organizada de manera semi-estructurada, y en la primera parte se consulto acerca de las costumbres de los entrevistados respecto de la compra de pescado, así como sus motivos, el tipo de pescado y el formato. También se consultó respecto de los atributos evaluados durante la compra y consumo de pescado en general y en particular del consumo de Tilapia, así como aspectos relativos al formato de compra, tipo de productos comprados, presentación, tipo de pescado comprador, atributos de calidad relevantes y finalmente la disposición a comprar y consumir un tipo de pescado no conocido. La información recolectada en esta etapa se empleó para la confección de un cuestionario aplicado en la segunda etapa de la investigación.

El cuestionario diseñado en la segunda etapa incluyó preguntas de filtro, para asegurar que la muestra correspondiese a habitantes del Gran Santiago y que fuesen consumidores regulares de pescado. A nivel de atributos, se incluyeron preguntas basadas en escala hedónica de cinco puntos, con el objeto de evaluar el nivel de importancia que el entrevistado asignada a diferentes atributos de calidad consultados. Para controlar potenciales errores de redacción, de comprensión de las preguntas, o de la estructura del cuestionario, se aplicó un estudio piloto con 15 encuestas.

El cuestionario final fue aplicado a una muestra de 385 personas, la que fue calculada de acuerdo a la fórmula para el muestreo desde poblaciones infinitas (Briones, 
2002), donde $\mathrm{n} 0=(\mathrm{Z} 2 \times \mathrm{P} \times \mathrm{Q}) / \mathrm{E} 2$. Asumiendo un valor de $\alpha=0,05$, con una distribución $Z$ de 1,96 , se obtuvo que $\mathrm{n} 0=(1,962 \times 0,5 \times 0,5) / 0,052$ 384. Para estimar una proporción en la muestra se utiliza el Teorema de o Principio de Pareto (Carot, 1998), donde se ordenan las comunas con mayor población a menor población, y se selecciona el $20 \%$ de las comunas más pobladas, de un total de 34 comunas, se seleccionan 7 , donde estas 7 son las utilizadas geográficamente para realizar las encuestas, logrando una representatividad de un $80 \%$ de las comunas del Gran Santiago. Las 7 comunas seleccionadas fueron Puente Alto, Maipú, La Florida, Las Condes, San Bernardo, Peñalolén y Santiago Centro, en las cuales se realizaron 84, 80, 63, 43, 42, 37 y 34 encuestas respectivamente. Los datos obtenidos fueron analizados mediante el programa de análisis estadístico SPSS, con el fin de generar información de frecuencia, media aritmética y análisis de varianza (ANOVA).

En la tercera etapa de la investigación se empleó un panel sensorial de tipo piloto, debido a lo desconocido del producto evaluado para los consumidores estudiados, para lo cual se seleccionaron 50 panelistas distribuidos de manera equitativa entre hombres $\mathrm{y}$ mujeres. El panel se estructuró a partir de una un cuestionario basado en información recopilada en las entrevistas en profundidad. El panel se realizó en dos etapas, en la primera se solicitó al panelista contestar un breve cuestionario, de modo de filtrar panelistas con discapacidad para percibir color y sabor, debido a razones físicas o congénitas, así como por el consumo de tabaco $\mathrm{u}$ otras sustancias capaces de afectar la percepción sensorial.

Se evaluaron tres productos, filete crudo fresco (Figura 1), filete cocido (Figura 2) y filete asado (Figura 3). El filete fresco se compró en una cadena de supermercados del Gran Santiago y fue transportado al laboratorio de análisis sensorial bajo condiciones de frío ( $0^{\circ}$ C. a $4^{\circ}$ C. $)$. La escala de evaluación proporcionada a los panelistas fue de tipo hedónica de cinco puntos, con el objeto de medir el nivel de agrado de los atributos de cada producto y mediante una prueba de aceptación se evaluó si comprarían el producto en formato fresco y si les gusta o no les gusta el filete de Tilapia asado y cocido. Para realizar el análisis se proporcionó a cada participante una muestra a evaluar, de $30 \mathrm{~g}$, de acuerdo a lo sugerido por Pedrero y Pangborn (1989). Las muestras fueron codificadas como A1 para filete fresco, A2 para filete cocido y B2 para filete asado, siendo la muestra A1 presentada en primer lugar para todos los panelistas, mientras que las muestras A2 y B2 fueron presentadas de manera aleatoria.

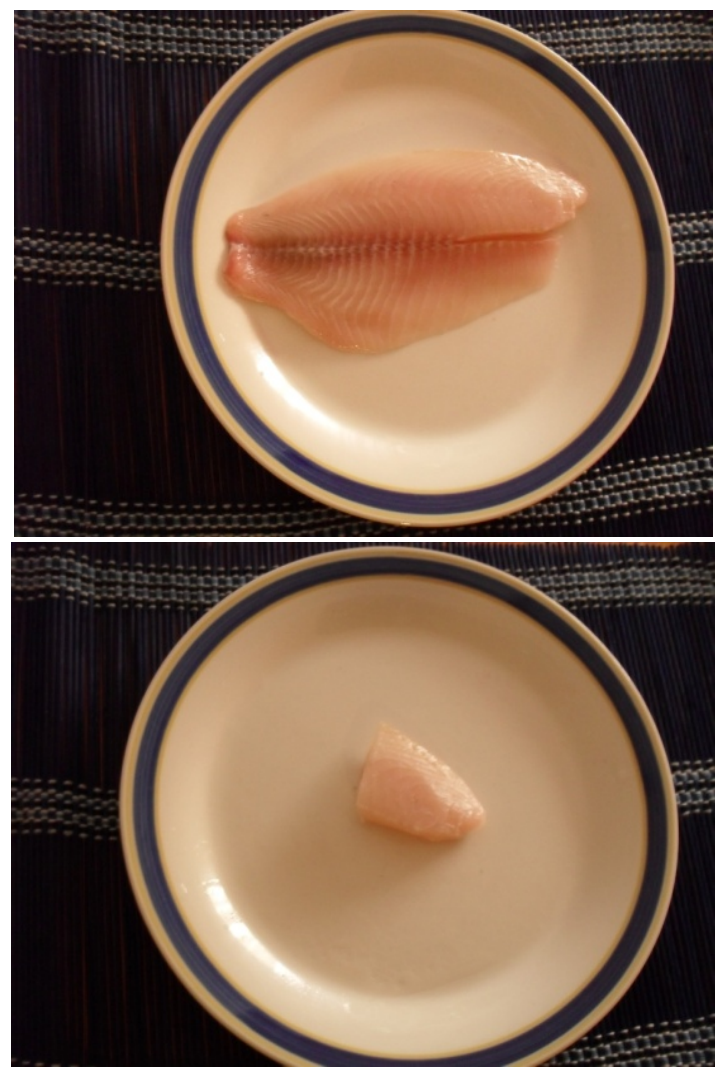

Imagen 1, Fotografías de filete y muestra fresca (cruda) de Tilapia. Arriba: Filete típico de tilapia (110 g.). Abajo: Corte de $30 \mathrm{~g}$. De filete de Tilapia formato fresco. 

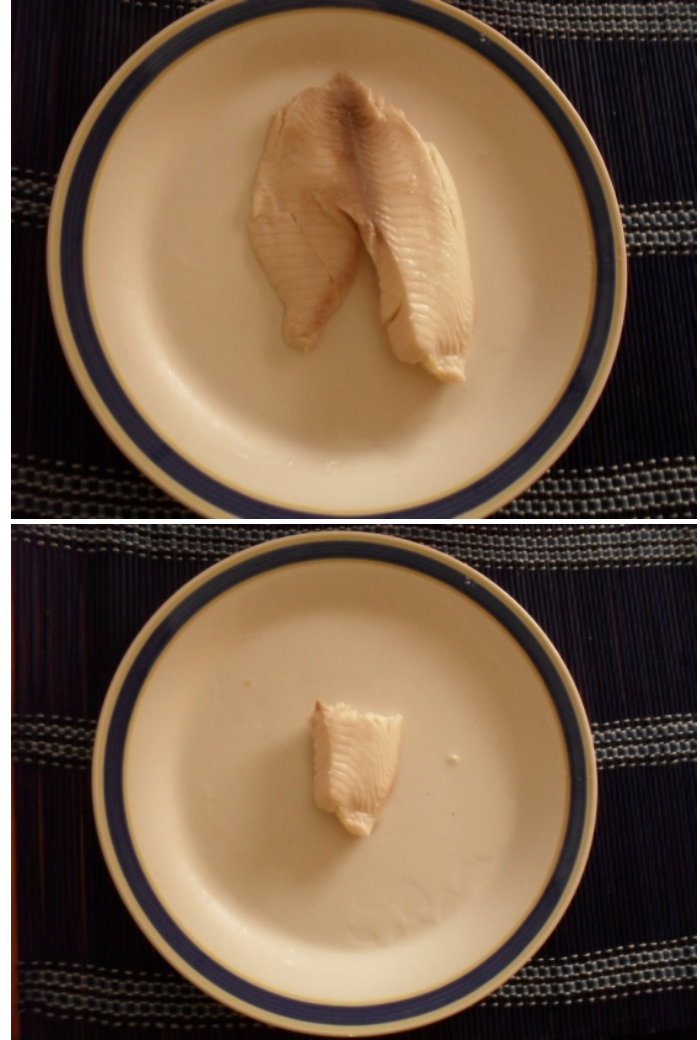

Imagen 2, Fotografías de filete y muestra cocida de Tilapia. Arriba: Filete típico de Tilapia (110 g.), abajo: Corte de $30 \mathrm{~g}$. De filete de Tilapia cocida.

Se evaluaron los atributos, color, olor textura y precio, para filete fresco, donde cada panelista podía, tocar, oler y observar la muestra. Además, incluía una evaluación del precio de mercado del producto en un supermercado particular de la Región Metropolitana, el cual consistía en $\$ 8.490$ por kilogramo.

Para la preparación de las muestras de filete cocido se añadió $1 \mathrm{~g}$ de sal por cada filete de $100 \mathrm{~g}$. y $14 \mathrm{ml}$. de aceite, los que se cocinaron $300 \mathrm{cc}$ de agua durante $5 \mathrm{a}$ 6 minutos, mientras que las muestras de filete asado, fueron condimentadas de idéntica manera, y luego asadas a una temperatura de $320^{\circ} \mathrm{C}$ en un horno eléctrico, durante 10 minutos aproximadamente. Adicionalmente, cada evaluador realizo una evaluación visual de filetes de $110 \mathrm{~g}$, similares a aquellos desde donde se extrajeron las muestras de $30 \mathrm{~g}$.
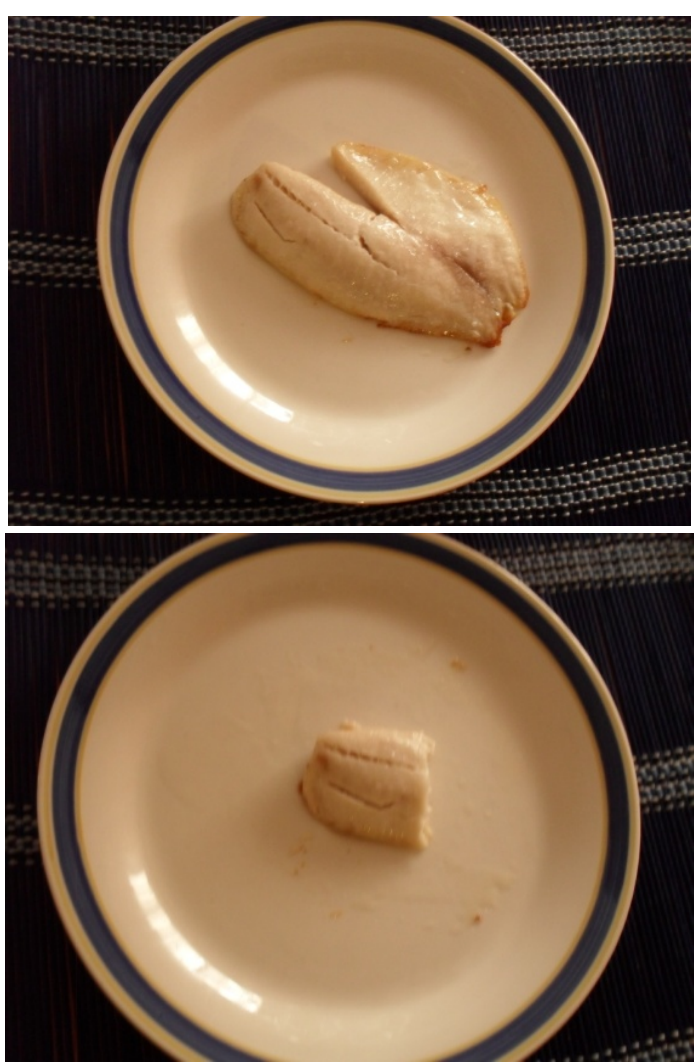

Imagen 3, Fotografías de filete y muestra asada de Tilapia. Arriba: Filete típico de Tilapia (110 g.), abajo: Corte de $30 \mathrm{~g}$. De filete de Tilapia asada.

Los datos fueron tabulados y analizados mediante el programa de análisis estadístico SPSS (Monegal, 1999), con el fin de generar gráficos de frecuencia, cálculo de media aritmética y análisis de varianza (ANOVA), de modo de evaluar la aceptación de los productos antes mencionados.

\section{Resultados}

Encuestas

A nivel de motivaciones para comprar pescado, los motivos vinculados a la alimentación saludable y el consumo de vitaminas y diversos nutrientes, son los más valorados por los consumidores entrevistados, seguidos por motivos vinculados al sabor y el conocimiento de la forma en que el pescado comprado debe ser preparado (Figura 4). 


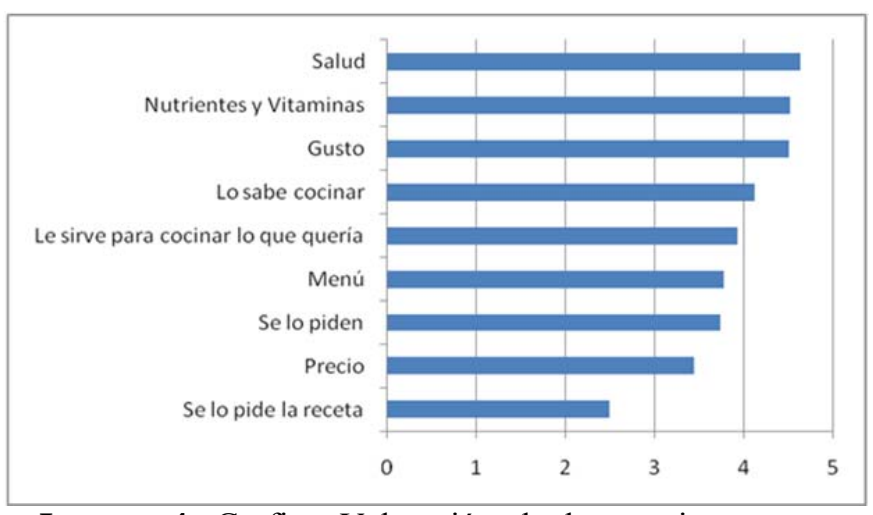

Imagen 4, Grafico Valoración de los motivos para comprar pescado.

A nivel de preferencias generales y hábitos de consumo de pescados de los entrevistados, los datos recopilados mediante el cuestionario indican que la mayor parte de los entrevistados prefieren comprar pescado fresco sin envasar $(68,2 \%)$, seguido por pescado enlatado (Tabla 1). Respecto de la presentación del pescado, de entre los entrevistados que indicaron comprar ellos mismos el producto (165), estos están divididos en proporciones similares, donde el $50,9 \%$ prefería comprar el pescado entero, y el 49,1\% compraba directamente el producto fileteado. Se destaca que quienes compran pescado entero, lo piden de distintas formas, por lo que el formato final es variable (Tabla 2).

Tabla 1, Preferencias de envasado

\begin{tabular}{c|cc}
\hline Ítem & Frecuencia & Porcentaje \\
\hline $\begin{array}{c}\text { Pescado a sin envasar (a } \\
\text { granel) }\end{array}$ & 262 & 68,2 \\
$\begin{array}{c}\text { Pescado enlatado } \\
\text { Pescado envasado en }\end{array}$ & 89 & 23,2 \\
Bandejas & 10 & 2,6 \\
Pescado envasado en Caja & 3 & 0,8 \\
Pescado envasado en Bolsas & 6 & 1,6 \\
Pescado envasado al Vacío & 13 & 3,4 \\
Pescado envasado en Papel & 1 & 0,3 \\
Total & 384 & 100,0 \\
\hline
\end{tabular}

Las preferencias de cortes del pescado comprado entero están dadas con un $30,9 \%$ por pescado en formato de primer corte, es decir, filete sin piel, sin espinas, sin cabeza, sin branquias, sin vísceras y $\sin$ escamas, seguido por el filete con piel y sin espinas, con un $17,6 \%$ de las preferencias y en tercer lugar con un $15,2 \%$ el filete con piel y espinas. Con relación a las preferencias por tipo de pescado comprado, estas se concentran fundamentalmente en dos especies Merluza (M. gayi) y Reineta (B. australis), las que concentran 38,8 y $26,8 \%$ de las preferencias de compra en primera instancia, respectivamente. A nivel de la valoración de atributos del pescado fresco durante la compra (Figura 5), olor y color son más valorados que textura y precio, mientras que para el pescado cocinado, sabor y aroma son más valorados por los entrevistados (Figura 6).

Tabla 2, Preferencias respecto de la presentación del producto

\begin{tabular}{|c|c|c|}
\hline Ítem & Frecuencia & Porcentaje \\
\hline \multirow{5}{*}{$\begin{array}{c}\text { Filete, con piel y espinas } \\
\text { Filete, con piel } \\
\text { Filete de primer corte } \\
\text { Filete, con espinas } \\
\text { Filete con piel, incluye } \\
\text { cabeza }\end{array}$} & 25 & $15,2 \%$ \\
\hline & 29 & $17,6 \%$ \\
\hline & 51 & $30,9 \%$ \\
\hline & 3 & $1,8 \%$ \\
\hline & 5 & $3,0 \%$ \\
\hline \multirow{4}{*}{$\begin{array}{c}\text { Filete con espinas, incluye } \\
\text { cabeza } \\
\text { Entero con todo } \\
\text { Filete, con piel y espinas, } \\
\text { incluye cabeza } \\
\text { Entero, sin espinas ni } \\
\text { cabeza }\end{array}$} & 4 & $2,4 \%$ \\
\hline & 11 & $6,7 \%$ \\
\hline & 20 & $12,1 \%$ \\
\hline & 2 & $1,2 \%$ \\
\hline \multirow{2}{*}{$\begin{array}{c}\text { Filete con espinas, incluye } \\
\text { branquias } \\
\text { Entero, sin branquias ni } \\
\text { escamas }\end{array}$} & 1 & $0,6 \%$ \\
\hline & 1 & $0,6 \%$ \\
\hline \multirow{2}{*}{$\begin{array}{l}\text { Entero, sin branquias ni } \\
\text { vísceras } \\
\text { entero sin escamas }\end{array}$} & 2 & $1,2 \%$ \\
\hline & 1 & $0,6 \%$ \\
\hline $\begin{array}{l}\text { Entero, sin branquias sin } \\
\text { vísceras ni cabeza }\end{array}$ & 4 & $2,4 \%$ \\
\hline \multirow{5}{*}{$\begin{array}{l}\text { Entero, sin branquias sin } \\
\text { escamas ni cabeza } \\
\text { Filete, incluye cabeza } \\
\text { Entero sin cabeza } \\
\text { Entero, sin vísceras sin } \\
\text { escamas ni cabeza } \\
\text { Total }\end{array}$} & 1 & $0,6 \%$ \\
\hline & 2 & $1,2 \%$ \\
\hline & 2 & $1,2 \%$ \\
\hline & 1 & $0,6 \%$ \\
\hline & 165 & $100,0 \%$ \\
\hline
\end{tabular}




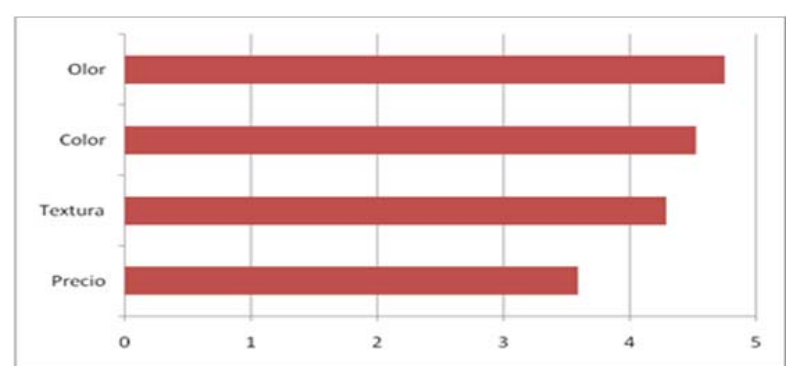

Imagen 5, Grafico Valoración de los atributos de calidad del pescado fresco durante la compra.

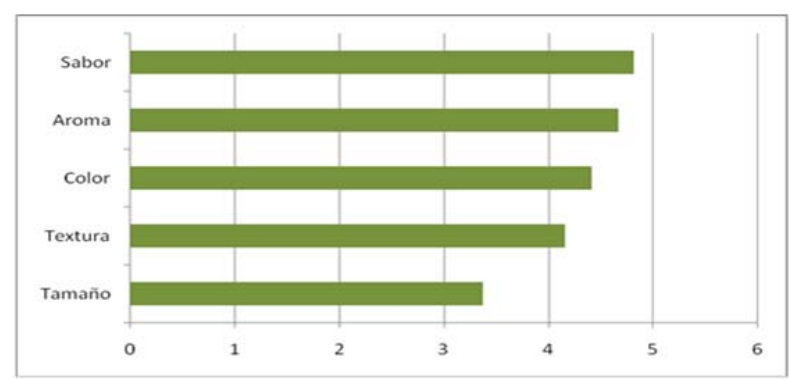

Imagen 6, Grafico Orden de importancia de los atributos de calidad del pescado cocinado.

\section{Análisis Sensorial}

Al evaluar los resultados de aceptabilidad de los atributos sensoriales del filete fresco de Tilapia, $64 \%$ y $24 \%$ de los participantes del panel piloto declara que el color les gusta y les gusta mucho respectivamente, mientras que en la evaluación de olor, $36 \%$ de los participantes indica que le gusta y que le gusta mucho. La evaluación mediante escala hedónica de 5 puntos indica que textura es el atributo mejor evaluado, seguido por color y olor (Figura 7), observándose que si bien los atributos olor y precio mantienen un nivel de significancia de 0,00 el atributo color mantiene un nivel de significancia de 0.03 , no obstante el atributo textura presenta un nivel de significancia de 0,06 (Tabla 3). Este valor de significancia puede ser explicado porque en el panel participaron consumidores que no realizan la compra de pescado en sus hogares, lo que genera un desconocimiento por parte de los mismos en la textura que tienen los filetes de pescado crudo, por lo que algunos de los asistentes al panel pudo elegir al azar su respuesta referente al nivel de agrado de la textura del producto.

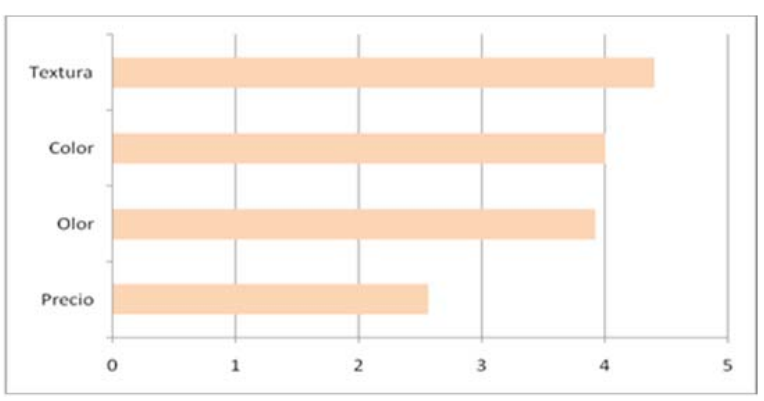

Imagen 7, Grafico Valoración de los atributos de calidad del pescado.

Tabla 3, ANOVA evaluación sensorial filete fresco de Tilapia.

\begin{tabular}{c|lcc}
\hline & & F & Sig. \\
Color & Inter-grupos & 3,516 &, 003 \\
Olor & Inter-grupos & 9,516 &, 000 \\
\hline Fresco & Inter-grupos & 3,175 &, 006 \\
\hline Precio & Inter-grupos & 10,849 &, 000 \\
\hline
\end{tabular}

En el caso de la evaluación de filete cocido de Tilapia, se observa que para el atributo color, $44 \%$ de los jueces indica que el color del producto les gusta, mientras que al $28 \%$ de le gusta mucho, y a un $20 \%$ le gusta poco. Además se debe considerar que el producto tiene una media de 3,7 que implica que en general el color del producto les gusta a los participantes (Figura 8). La evaluación del filete asado de Tilapia indica que tuvo una muy buena aceptación por los panelistas asistentes y al realizar un análisis por atributo, se observa que el atributo color mantiene una media de 4,4 (Figura 9), lo que implica que a los jueces en general les gustó el color de la Tilapia asada, manteniendo una concentración del $52 \%$ de los jueces que les gustó mucho dicho atributo en el producto, seguido por un $40 \%$ de quienes indicaron que les gustó. 


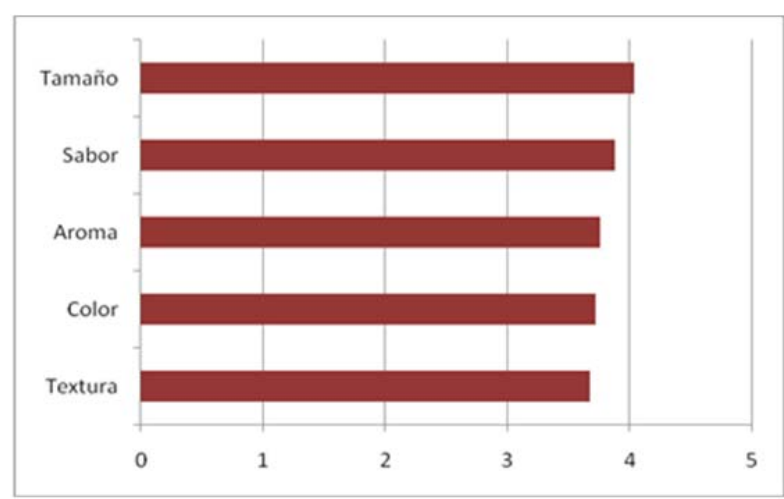

Imagen 8, Grafico Nivel de valoración de los atributos sensoriales de filetes cocidos evaluados.

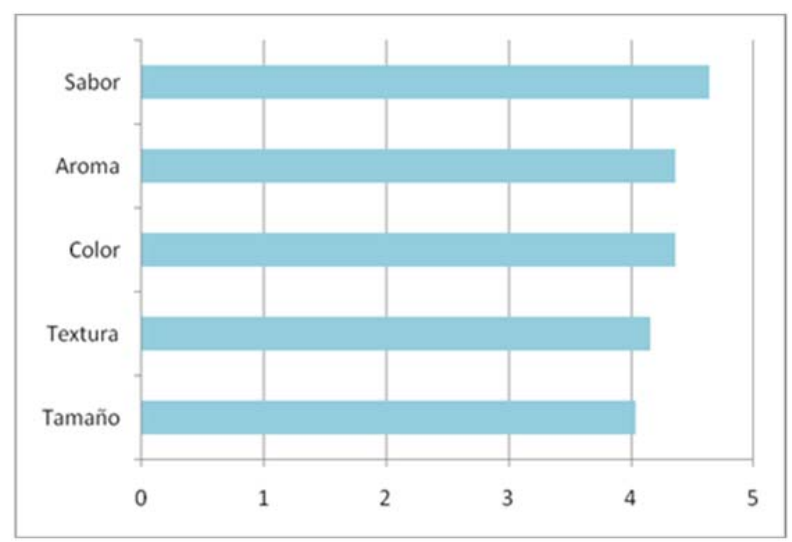

Imagen 9, Grafico Nivel de valoración de los atributos sensoriales de filetes asados evaluados.

\section{Concusiones}

Las motivaciones para la compra de Tilapia corresponderían a las mismas motivaciones que los consumidores declaran para la compra de pescados en general, es decir razones asociadas a la alimentación sana y aporte de nutrientes de este producto. Si bien los resultados exploratorios sugieren que los consumidores son reticentes de comprar un producto desconocido, la evaluación sensorial indica que los atributos del producto son altamente aceptables para los panelistas, con un valor mínimo de 4 puntos en una escala hedónica de 5 puntos.

La evaluación de los atributos sensoriales es, aparentemente, dependiente de la forma en que este es preparado. Se destaca que el atributo tamaño del filete es altamente valorado en las muestras cocidas, mientras que en las muestras de Tilapia asada, este atributo es menos relevante en comparación a atributos tales como sabor aroma y color.

Considerando que las motivaciones para comprar pescado serían similares para los diferentes tipos de pescados comercializados en el Gran Santiago, tales como Merluza y Reineta, así como para especies recientemente introducidas, como Tilapia, se estima necesario profundizar estudios tendientes a detectar diferencias en la percepción de los atributos sensoriales de este producto, así como la percepción de la calidad vinculada a la preparación o valor culinario de este producto, el que aparentemente se presenta como una alternativa prometedora en la categoría de productos acuícolas.

\section{Referencia}

1. Carot, V.1998. Control estadístico de la calidad. Editorial UPV. España. 703p.

2. Castillo, L. 2005. Tilapia Roja 2005. Cali, Colombia. $116 \mathrm{p}$.

3. Castillo, L. 2010. Estado actual de la Tilapia Junio 2010.Cali, Colombia. 26p.

4. Corpei. 2005. Resumen de Estudio: Subsector Tilapia. Consultado el día 25 de Septiembre de 2010. Disponible en: http://www.corpei.org/archivos/file/Ecuador\%20In vest/RESUMEN\%20SUBSECTOR\%20TILAPIA. pdf.

5. Maradiegue, R.; Farro, E.; Escala, J.; Yi, D. 2005. Planteamiento estratégico para la producción y comercialización de Tilapias. Tesis Magister en Adm. De Negocios. Universidad Católica del Perú. Surco, Perú. 246 p.

6. Monegal, M. 1999. Introducción al SPSS: Manipulación de datos y estadística descriptiva. Edicions de la Universitat de Barcelona. Barcelona, España.170 p. 
7. Morales, G.; Blanco, L.; Arias, M. y Chaves C. 2004. Evaluación de la calidad bacteriológica de tilapia fresca (Oreochromis niloticus) proveniente de la Zona Norte de Costa Rica. ALAN v.54 n.4 Caracas dic.

8. Pedrero, D. y Pangborn, R. 1989. Evaluación sensorial de los alimentos. Métodos analíticos. Editorial Alhambra Mexicana. 251 p. 